\title{
High Density 3D Mapping and Ablation of Complex Cardiac Arrhythmias: Our Experience in NICVD.
}

\author{
Md. Mohsin Hossain ${ }^{1}$, Md. Mustafizur Rahman², Asif Zaman Tushar, Al-Mamun ${ }^{4}$, Md. Nazmul Haq ${ }^{3}$, \\ Kanak Jyoti Mondol ${ }^{5}$
}

\begin{abstract}
:
Background: Catheter ablation can be curative in patients with drug-refractory tachyarrhythmias. 3D electro anatomical mapping (EAM) is an established tool facilitating catheter ablation. This system is particularly valuable for mapping complex arrhythmias, which provide excellent assistance to catheter navigation, reduces fluoroscopy exposure, and also allow for the accurate placement of catheters. The Rhythmia Mapping System (RMS, Boston Scientific) is a novel system that allows for ultra-fast, high-density 3D mapping.
\end{abstract}

Aim of this Study: The aim of this study was to find out the result of a high-density 3D mapping for the ablation of complex Cardiac Arrhythmias and to share our experiences.

Methods: A total number of 44 patients of different tachyarrhythmias were scheduled for catheter ablation by Rhythmia Mapping System in National Institute of Cardiovascular Diseases, Bangladesh from 3rd February'2018 to 18th July'2019. During and after, the procedure all the cases were evaluated for different procedure parameters, acute success and in-hospital success.

Results: Among the patients (28/44 male) 13 (25.55\%) cases were atrial fibrillation, $6(16.64 \%)$ cases were atrial flutter, $6(16.64 \%)$ cases were atrial tachycardia, 2 (4.55\%) cases were ventricular tachycardia, $11(25 \%)$ cases were PVC and $6(16.64 \%)$ cases were accessory pathway. The mean age was $38 \pm 4.5$ years. In $25(56.82 \%)$ of tachyarrhythmia patients, the mechanism was macro reentry/micro reentry, while in $19(43.18 \%)$ cases the mechanism was increased automaticity. In all cases, the tachycardias were adequately mapped \& proper identification of focus was done during the index procedure with the ultra-high density 3-D Rhythmia Mapping System (RMS). These all were successfully terminated by radiofrequency ablation, except one, which was one of the two cases of Ventricular tachycardia. With this system our study samples had a success rate of $98 \%$ with arrhythmia elimination. In patients of atrial fibrillation, all 4 pulmonary veins isolation were done. The mean mapping time was $28.6 \pm 17$ minutes, and the mean radiofrequency ablation time to arrhythmia termination was $3.2 \pm 2.6$ minutes. During our study only two out of $\mathbf{4 4}$ patients developed complications. One of the patients with atrial fibrillation developed cardiac tamponade and the other patient with PVC originating from Aortic cusp developed ischemic stroke. Fortunately, they were both managed accordingly. During hospital discharge, all the patients were free of tachyarrhythmia and were in sinus rhythm.

Conclusions: This new automated ultrahigh-resolution mapping system allows accurate diagnosis of tachyarrhythmia circuits. Ablation of the focus resulted in high acute success.

Keywords: Ultra-High density, 3D mapping, Cardiac Arrhythmias, Rhythmia Mapping System.

(Bangladesh Heart Journal 2021; 36(2): 98-104)

1. Professor of Cardiology, Department of Cardiac Electrophysiology, NICVD, Dhaka, Bangladesh.

2. Assistant Professor, Department of Cardiac Electrophysiology, NICVD, Sher-e-Bangla Nagar, Dhaka, Bangladesh.

3. Medical Officer, Department of Cardiac Electrophysiology, NNICVD, Sher-e-Bangla Nagar, Dhaka, Bangladesh.

4. Junior Consultant. Department of Cardiac Electrophysiology, NICVD, Sher-e-Bangla Nagar, Dhaka, Bangladesh.

5. Resident, Department of Cardiac Electrophysiology, NICVD, Sher-e-Bangla Nagar, Dhaka, Bangladesh.

Corresponding author: Dr. Md. Mustafizur Rahman Palash, Assistant Professor, Department of Cardiac Electrophysiology, National Institute of Cardiovascular Diseases \& Hospital, Sher-e-Bangla Nagar, Dhaka, Bangladesh. Email: drmmrpalash@gmail.com,contact no: +8801816641767 .

DOI: https://doi.org/10.3329/bhj.v36i2.56035

Copyright $\odot 2017$ Bangladesh Cardiac Society. Published by Bangladesh Cardiac Society. This is an Open Access articles published under the Creative Commons Attribution-NonCommercial 4.0 International License (CC BY-NC). This license permits use, distribution and reproduction in any medium, provided the original work is properly cited and is not used for commercial purposes. 


\section{Introduction:}

Catheter ablation can be curative in the patients of drug refractory tachyarrhythmia. 3D electro anatomical mapping (EAM) is an established tool facilitating catheter ablation. This system is particularly valuable for mapping complex arrhythmias, which provide excellent assistance to catheter navigation, reduces fluoroscopy exposure and also allow for the accurate placement of catheters. The Rhythmia Mapping System (RMS, Boston Scientific) is a novel system that allows for ultra-fast, high- density $3 \mathrm{D}$ mapping.

Contemporary High-Resolution Mapping Systems are fit for obtaining and annotating multiple Electrograms (EGMs), which are handled via mechanized calculations to produce actuation and substrate guides to help and guide removal methodology. All the more as of late, a novel Ultra-High Density (UHD) Electroanatomic Mapping System empowers fast programmed procurement of HighResolution Maps through a 64 Pole Basket Array Catheter with little and firmly dispersed ELECTRODES in mix with a committed Mapping Platform (Boston Scientific, Marlborough, MA, USA). The remarkable qualities of fast and programmed procurement of Maps with High Spatiotemporal Resolution, without the requirement for broad manual comment, has been assessed in PreClinical and beginning experience studies. ${ }^{1-3}$ Furthermore, various human examinations announced information in a wide scope of genuine Clinical Settings including the utilization of Mapping for both Atrial and Ventricular Arrhythmias ${ }^{4-9}$ Although a large portion of these investigations have shown that the System is protected, effectual and Clinically helpful in explicit settings, to date there has been no planned investigation to evaluate the intense wellbeing, intense viability and Clinical utilization of the System on a wide range of various Arrhythmias to direct Ablation in certifiable Clinical Practice. Contemporary High-Resolution Mapping Systems are capable of acquiring and Annotating Multiple Electrograms (EGMs), which are processed by automated algorithms to generate activation and substrate maps to support and guide ablation procedures. In this study, our main objective is to share our experiences regarding find out the result of a high-density 3D mapping for the ablation of complex Cardiac Arrhythmias. Also, the research will provide a comprehensive insight into the overall significance of topic of our study.

\section{Method:}

The study was a prospective, observational and single centered study conducted using purposive sampling technique. The objective was to assess the acute safety, procedural success, and the utility of a novel RMS (Boston Scientific) and to share our experience. The RMS system was paired with a catheter with small and closely spaced electrodes in patients who underwent an ablation procedure in a clinical setting. A total number of 44 patients of different tachyarrhythmias were scheduled for catheter ablation by Rhythmia Mapping System in National Institute of Cardiovascular Diseases and hospital, Dhaka, Bangladesh from 3rd February'2018 to 18th July'2019. During and after, the procedure all the cases were evaluated for different procedure parameters, acute success and in hospital success.

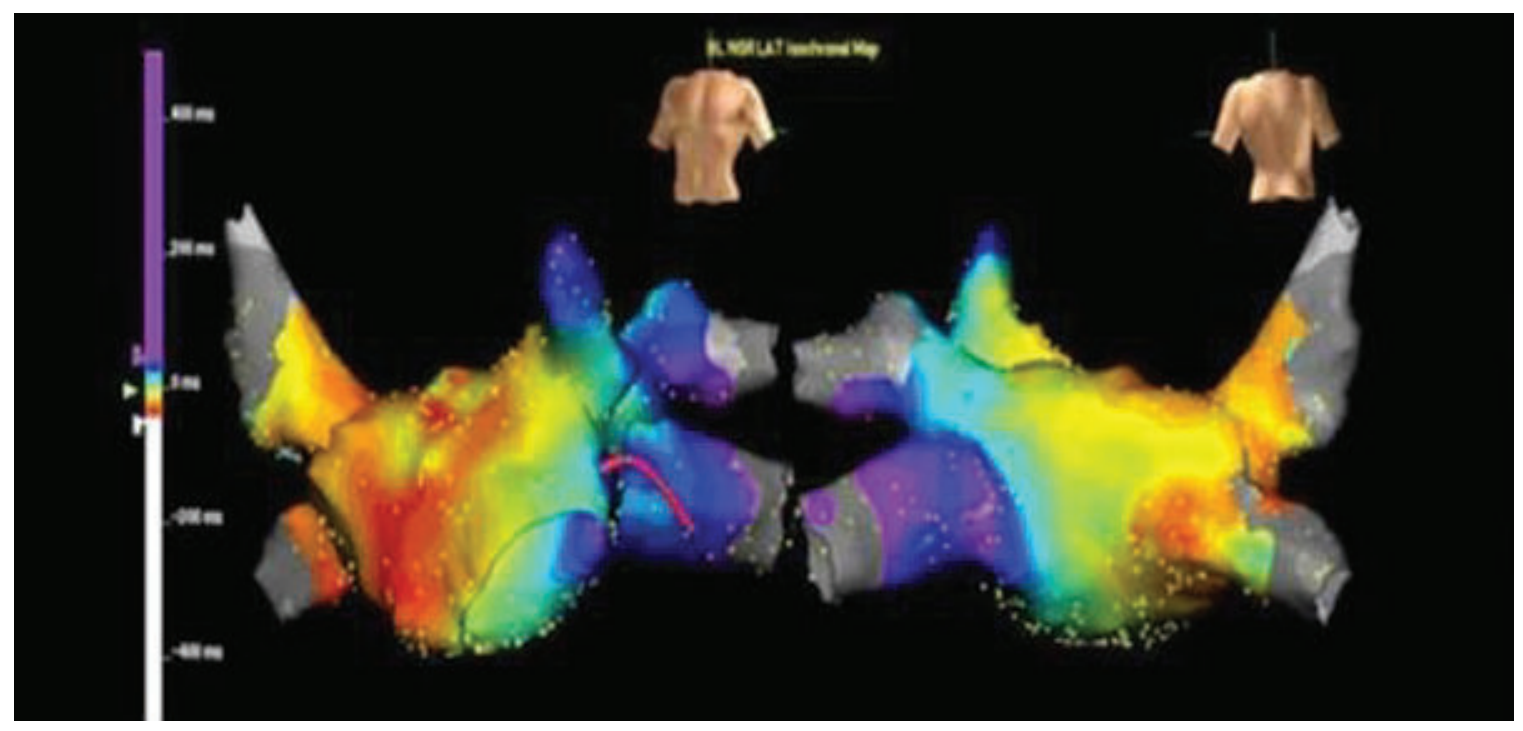

Fig.-1: 3D Mapping with RMS. 
All 44 patients enrolled in the study with conscious consent. The undergoing index ablation procedure were required to use the catheter in conjunction of the Rhythmia Mapping System (RMS). Any commercially available ablation catheter was used in the study with the discretion of the attending physicians. The primary effectiveness endpoint was the acute procedural success for mapping and ablation of the primary clinical cardiac arrhythmia. A procedure was considered acutely successful if all the following criteria were met;

I. Ability to map the primary arrhythmia (ongoing) or its electro-anatomical substrate with the RMS,
II. Completion of the indispensable ablation lesions,

III. Arrhythmia cessation, (in applicable cases), and

IV. Ablation substantiation through a documented validated technique.

Procedures where the mapping catheter system entered the cardiac chamber, but no electroanatomic maps were created for either diagnosis or validation purposes. These were categorized as an attempt, regardless of whether ablation was achieved or not. Procedures with at least one utilized map for diagnosis or validation defined the treatment cases. Success with validation in treatment cases was required to count a success for the primary effectiveness endpoint.

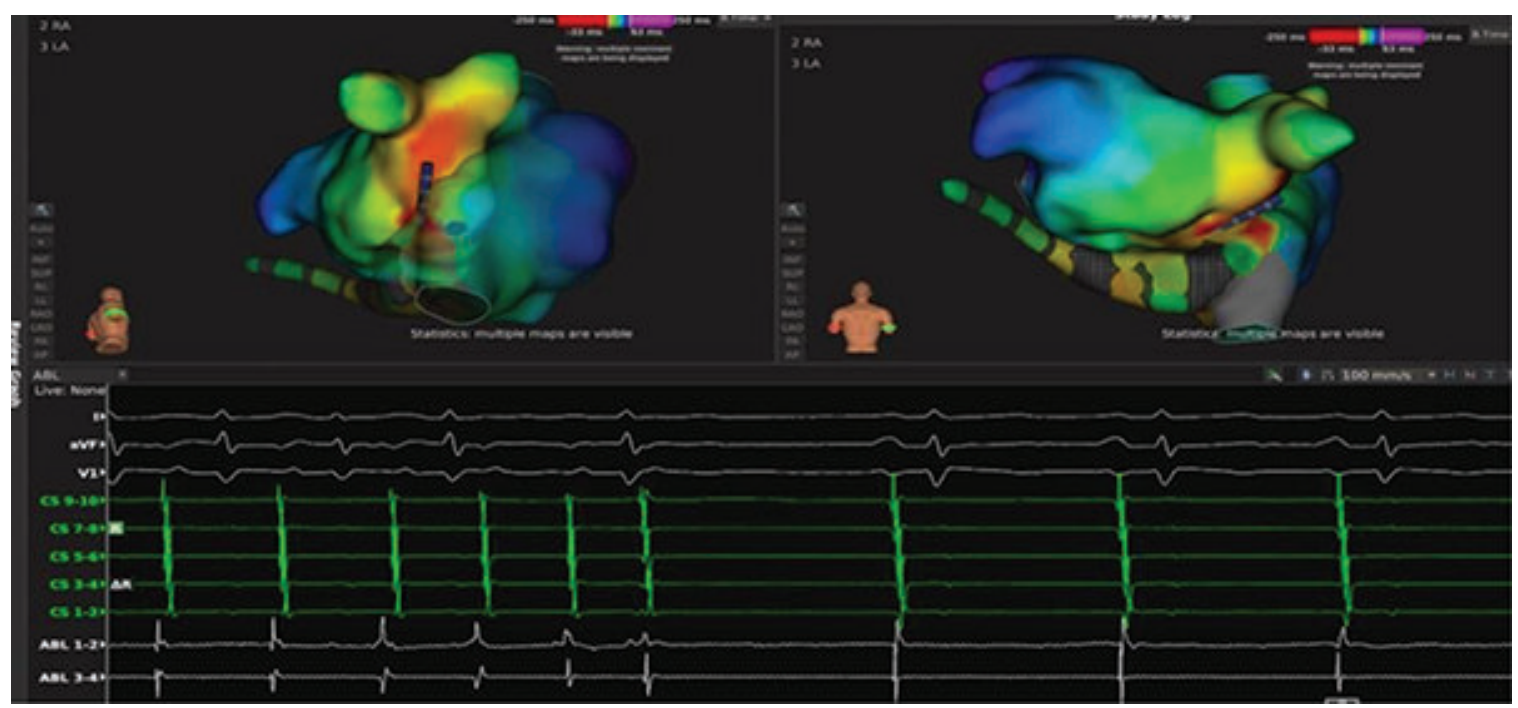

Fig.-2: $3 D$ mapping of the RA \&LA was done which revealed Atrial tachycardia originated from RA appendage. Termination of tachycardia by successful ablation.
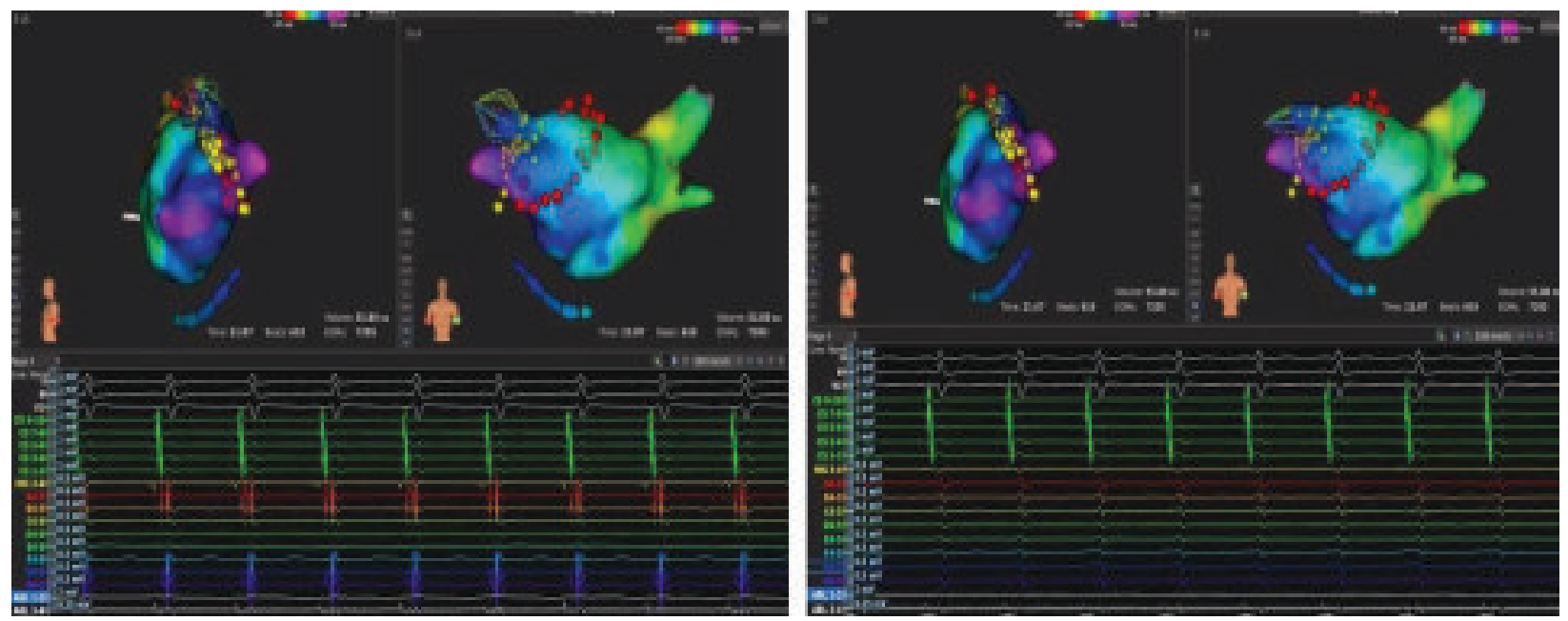

Fig.-3: 3D mappping of the left \& right, superioir \& inferior pulmonary veins and isolation was done in case of atrial fibrillation. The pictures showing left superior pulmonary vein (LSPV) isolation before \& after ablation by the absence of PV potential. 


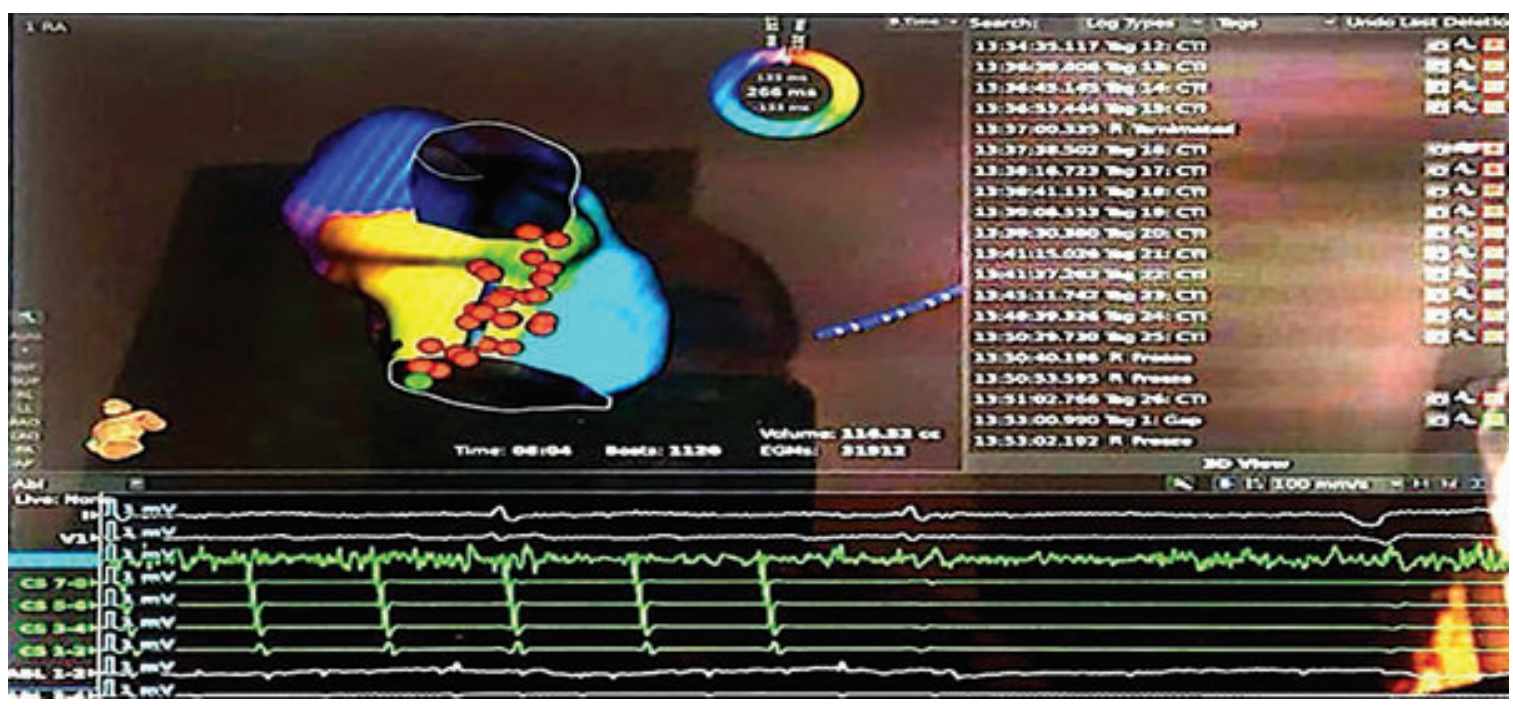

Fig.-4: 3D mapping of the Right atrium which revealed counterclockwise activation of the atrial flutter. Successful termination of atrial flutter by CTI ablation.
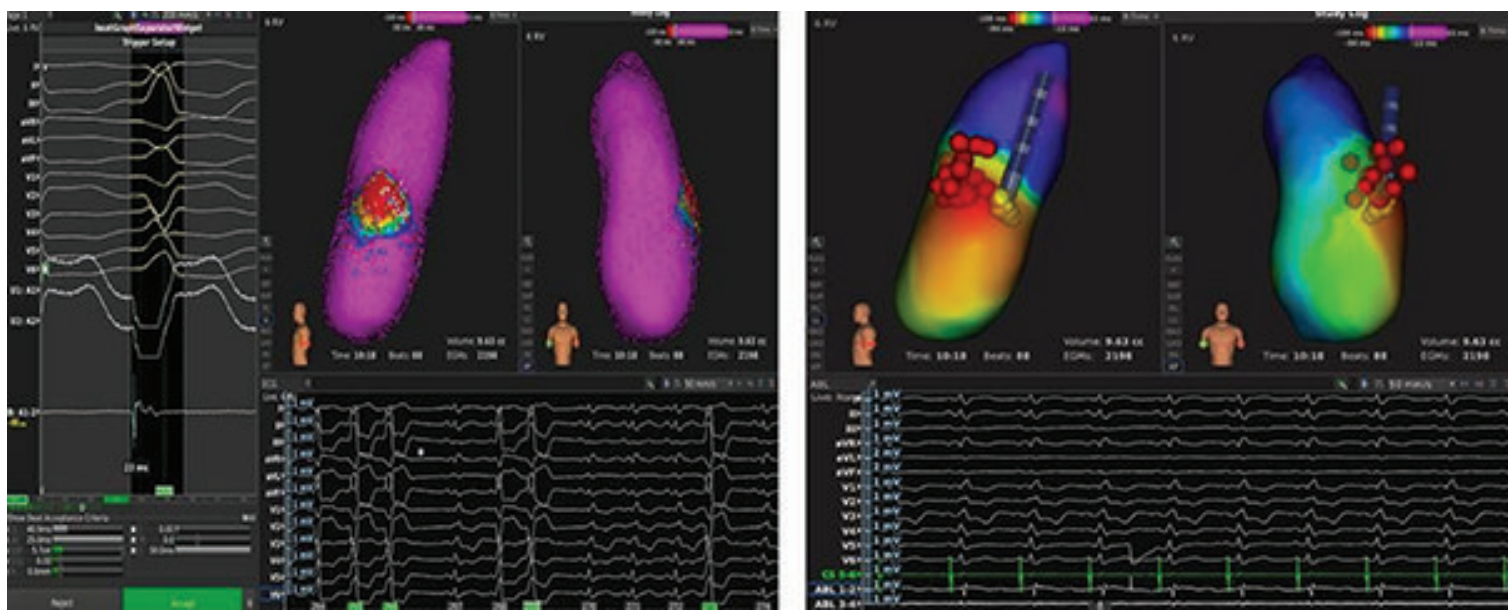

Fig.-5: 3D mapping of the right ventricle \& RVOT by RMS which revealed PVC originated from anterior RVOT. Right side of the picture showing termination of PVC by successful ablation.

The primary safety endpoint included all serious adverse events and study devices related adverse events within 30 days after the procedure. Seriousness and relationship with the procedure/device were determined by the investigator at each study site. In all cases of the study, the tachycardias were adequately mapped \& proper identification of focus was done during the index procedure and successfully terminated by radiofrequency ablation. In patients of atrial fibrillation all 4 pulmonary veins isolation were done (Figure-3). However, on statistical analysis of the study, we primarily took on a ration-logical approach and used frequency distribution, mean, and standard deviation techniques to analyze the findings of our study. All 44 samples were informedly participated in the study with the means of a consent form as well as with a verbal agreement.

\section{Result:}

The study was participated by 28 (63.64\%) male which were the majority samples of the study. The remaining $36.36 \%$ were of female gender (Figure-6).

Majority of the sample had a clinical history of atrial fibrillation. Few of the samples had the clinical history of multiple cardiac arrhythmia symptoms which in reflected in the Table-1 below. The mean age of the samples was 38 with the standard deviation of 4.5 as shown in Table-l. 


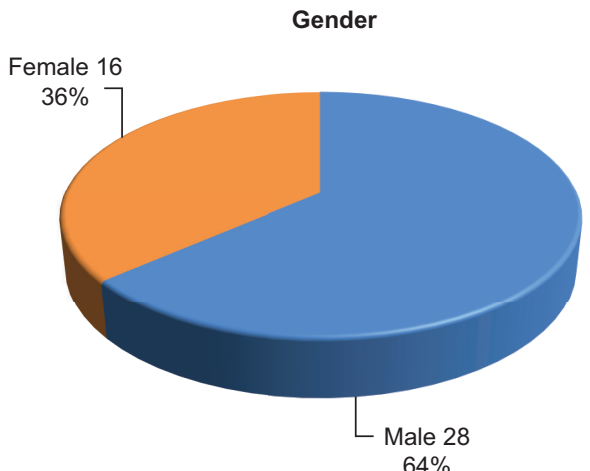

Fig.-6: Gender Distribution. ( $n=44)$
Table-I

Demographic Distribution and baseline characteristics Among the Samples $(n=44)$

\begin{tabular}{ll}
\hline Demographic Description & \\
Age (years) & $38 \pm 4.5$ \\
Male, N (\%) & $28(63.64 \%)$ \\
Female, N (\%) & $16(36.16 \%)$ \\
Hypertension & $5(11.36 \%)$ \\
Diabetes Mellitus & $02(4.54 \%)$ \\
LV Function (LVEF\%) & $64 \pm 05 \%$ \\
\hline
\end{tabular}

Table-II

Clinical History of Arrhythmia Among the Samples. ( $n=44)$ And Results

\begin{tabular}{lccccc}
\hline Clinical Arrhythmia History & Number & Percentage $\%$ & \multicolumn{2}{c}{ Outcome } & Complications \\
\cline { 4 - 5 } & & & Success & Failure & \\
\hline Ventricular tachycardia & 2 & $4.55 \%$ & 01 & 01 & 0 \\
Atrial fibrillation & 13 & $25.55 \%$ & 13 & 0 & 01 \\
Atrial tachycardia & 6 & $16.64 \%$ & 06 & 0 & 0 \\
Atrial flutter & 6 & $16.64 \%$ & 06 & 0 & 0 \\
PVC & 11 & $25.00 \%$ & 11 & 0 & 01 \\
Accessory Pathways & 6 & $16.64 \%$ & 06 & 0 & 0 \\
\hline Total & 44 & & $43(98 \%)$ & $01(2 \%)$ & $02(4.5 \%)$ \\
\hline
\end{tabular}

Table-III

Complications \& its frequency during our procedure.

\begin{tabular}{lc}
\hline Complications & Frequency \\
\hline 1. Cardiac Tamponade & $1(2.27 \%)$ \\
2. Ischemic stroke & $1(2.27 \%)$ \\
\hline Total & $2(4.54 \%)$ \\
\hline
\end{tabular}

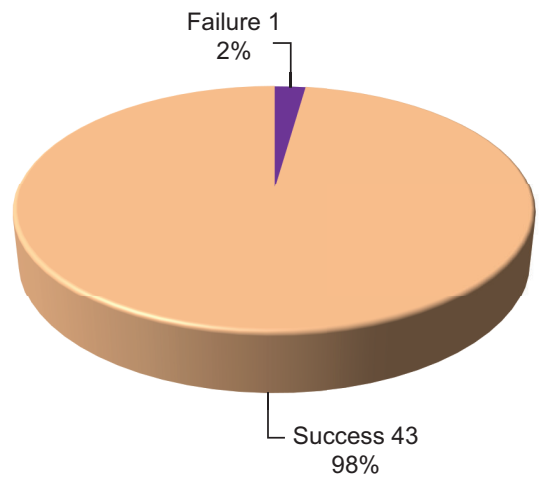

Fig.-7: Success rate of 3-D mapping \& ablation at our Centre
In $25(56.82 \%)$ of tachyarrhythmia patients the mechanism was macroreentry/microreentry, while in 19 $(43.18 \%)$ cases the mechanism was increased automaticity as shown in Figure-7.

The mean mapping time was $28.6 \pm 17$ minutes, and the mean radiofrequency ablation time to arrhythmia termination was $3.2 \pm 2.6$ minutes.

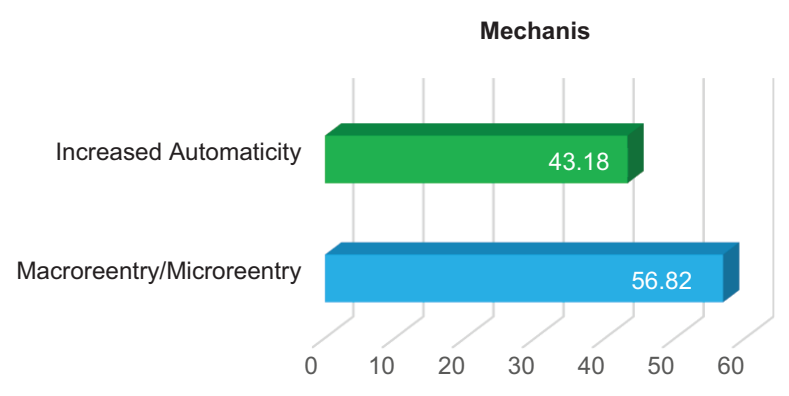

Fig.-8: Tachyarrhythmia Mechanism. $(n=44)$ 
Table-IV

Mapping Description.

\begin{tabular}{lc}
\hline Mapping Description & Mean \pm SD \\
\hline Mapping time (in minutes) & $28.6 \pm 17$ \\
$\begin{array}{l}\text { Radiofrequency ablation time to } \\
\text { arrhythmia termination (in minutes) }\end{array}$ & $3.2 \pm 2.6$ \\
\hline
\end{tabular}

\section{Discussion:}

This is the first prospective, single-centred study in Bangladesh to perform systematic data collection in order to describe clinical use of a UHD mapping technology as used in different arrhythmias. Previous studies published on this topic included patients, ${ }^{3,5-7}$ focused on specific mapping capabilities or concentrated on specific arrhythmia types. ${ }^{8}$ Moreover, these studies demonstrated the clinical advantages in the management of particular cases such as complex atrial ${ }^{9}$ or ventricular tachycardias (VTs). In our study, we used A total number of 44 patients of different tachyarrhythmia were scheduled for catheter ablation by Rhythmia Mapping System in National Institute of Cardiovascular Diseases, Bangladesh during 3rd February'2018 to 18th July'2019. During and after, the procedure all the cases were evaluated for different procedure parameters, acute success and in hospital success. the patients (28/44 male) $13(25.55 \%)$ cases were atrial fibrillation, 6 $(16.64 \%)$ cases were atrial flutter, $6(16.64 \%)$ cases were atrial tachycardia, $2(4.55 \%)$ cases were ventricular tachycardia, 11 (25\%) cases were PVC and 6 (16.64\%) cases were accessory pathway. Mean age was $38 \pm 4.5$ years. In all cases, the tachycardias were adequately mapped \& proper identification of focus was done during the index procedure. These were successfully terminated by radiofrequency ablation, except one, which was one of the two cases of Ventricular tachycardia. In our study, $25(56.82 \%)$ of tachyarrhythmia patients the mechanism was macroreentry/microreentry, while in 19 (43.18\%) cases the mechanism was increased automaticity.

In patients of atrial fibrillation all 4 pulmonary veins isolation were done. The mean mapping time was 28.6 \pm 17 minutes, and the mean radiofrequency ablation time to arrhythmia termination was $3.2 \pm 2.6$ minutes. The mapping time coincides with the approximate timings of few other studies, ${ }^{5,8-10}$ and proves the high efficiency of the RMS. With this system our study samples had a success rate of $98 \%$ with arrhythmia elimination. During our study only two out of 44 patients developed complications. The patient with atrial fibrillation developed cardiac tamponade and the patient with PVC originating from Aortic cusp developed ischemic stroke. Fortunately, they were both managed accordingly. The success rate is not so overwhelming in studies that had more samples to work with, so it varies marginally from the facts and literature mentioned few other studies. 4,9,11 During hospital discharge all the patients were free of tachyarrhythmia and were in sinus rhythm.

\section{Conclusion:}

Different study evaluated the safety and acute effectiveness of using this novel UHD mapping system in a variety of clinical arrhythmias as standard of treatment throughout the world. Our study is the first real-world data acquired in Bangladesh that confirm that the new novel system performs well with a very low rate of complications. As we have studied on a limited sample within a short time-period, further studies are necessary to validate the facts mentioned in this research. However, with the given findings, we can conclude that the new automated ultrahigh resolution 3-D mapping system allows accurate diagnosis of tachyarrhythmia circuits and ablation of the focus, resulting in a high acute success.

\section{Reference:}

1. Nakagawa $H$, Ikeda $A$, Sharma $T$, Lazzara $R$, Jackman WM. Rapid high resolution electro anatomical mapping: evaluation of a new system in a canine atrial linear lesion model. Circ Arrhythm Electrophysiology 2012; 5:417-24.

2. Thajudeen A, Jackman WM, Stewart B, Cokic I, Nakagawa $\mathrm{H}$, Shehata $\mathrm{M}$ et al. Correlation of scar in cardiac MRI and high-resolution contact mapping of left ventricle in a chronic infarct model. Pacing Clin Electrophysiol 2015; 38:663-74.

3. Bollmann A, Hilbert S, John S, Kosiuk J, Hindricks G. Initial experience with ultra-high-density mapping of human right atria. J Cardiovasc Electrophysiol 2016; 27:154-60.

4. Yamashita S, Tokuda M, Isogai R, Tokutake K, Yokoyama K, Narui R et al. Spiral activation of the superior vena cava: the utility of ultra-high-resolution mapping for caval isolation. Heart Rhythm 2018; 15:193-200.

5. Luther V, Sikkel M, Bennett N, Guerrero F, Leong K, and Qureshi N et al. Visualizing localized reentry with ultra-high density mapping in iatrogenic atrial tachycardia: beware Pseudo-Reentry. Circ Arrhythm Electrophysiol 2017; 10:e004724.

6. Kosiuk J, Hilbert S, John S, Bertagnolli L, Hindricks G, Bollmann A. Preliminary experience with high- 
density electroanatomical mapping for ablation of atrial fibrillation-comparison of mini-basket and novel open irrigated magnetic ablation catheter in consecutive patients. Int J Cardiol 2017; 228:401-5.

7. Frontera A, Takigawa M, Martin R, Thompson N, Cheniti G, Massoullie' $G$ et al. Electrogram signature of specific activation patterns: analysis of atrial tachycardias at high-density endocardial mapping. Heart Rhythm 2018; 15:28-37.

8. Garcia-Bolao I, Ballesteros G, Ramos P, Mene'ndez $D$, Erkiaga A, Neglia $R$ et al. Identification of pulmonary vein reconnection gaps with high-density mapping in redo atrial fibrillation ablation procedures. Europace 2018; 20:f351-8.
9. Scheinman MM, Huang S. The 1998 NASPE prospective catheter ablation registry. Pacing Clin Electrophysiol 2000; 23:1020-8.

10. Ferrero de Loma-Osorio A, Gil-Ortega I, PedroteMart1'nez A. Spanish Catheter Ablation Registry. 13th Official Report of the Spanish Society of Cardiology Working Group on Electrophysiology and Arrhythmias. Rev Esp Cardiol (Engl Ed) 2014; 67:925-35.

11. Santangeli P, Di Biase L, Pelargonio G, Dello Russo A, Casella M, Sanchez J et al. Catheter ablation of atrial fibrillation: randomized controlled trials and registries, a look back and the view forward. J Interv Card Electrophysiol 2011; 31:69-80. 